\title{
Research Progress of LD Pumped Yb: YAG Solid-State Laser
}

\author{
Qun Liu \\ Department of Physics, Harbin University, Harbin, Heilongjiang, 150080
}

Keywords: LD Pumped Yb, YAG, Solid State Laser

\begin{abstract}
With the development and maturity of semiconductor technology, laser diodes have greatly improved in terms of power, conversion efficiency, wavelength expansion and operating life. The laser diode has the characteristics of high quantum efficiency, high reliability, long service life, good emission wavelength and laser medium absorption peak, and good laser output beam quality. This makes laser diode and pump technology combined to become an important breakthrough in laser pumping technology. The rapid development of pumped solid-state lasers has gradually become a trend.
\end{abstract}

\section{Introduction}

In 1959, the Soviet Union's Bassov academician proposed that the stimulated radiation could be realized in the Ga As material by the method of carrier injection. In 1960, the first ruby laser was born. Since then, the research of solid-state lasers has become the focus of laser research. At the time, Newman discovered that the Ga As diode could emit nearly $880 \mathrm{~nm}$ of radiation. He used a Ga As diode $880 \mathrm{~nm}$ radiation pumped argon-doped calcium tungstate crystal (Nd:Ca WO4) to obtain $1.06 \mu \mathrm{m}$ fluorescence. Then, in 1964, Keyes and Quist of the MIT Lincoln Laboratory in the United States successfully produced the world's first LD-pumped solid-state laser using Ca F2:U3+. During the same period, Ochs and Pankove also used a light-emitting diode array ((LED) pumped Ca F2:Dy2+ to obtain a quasi-continuous output at an injection power of $0.1 \mathrm{~W}$. However, due to the power and reliability of the LD under the conditions at the time The requirements of pumping light source are still not met. Although the advantages of LD instead of flash lamp as pump source are high, long life and compact structure, the related research reports are still few. In 1968, McDonnell Douglas The company's Ross successfully implemented the first Nd:YAG laser pumped with Ga As LD $(867 \mathrm{~nm})$ with Ga As LD $(867 \mathrm{~nm})$ pumped Nd3+:YAG in a low temperature environment of $170 \mathrm{~K}$. But this early LD output The power is too small to achieve effective DPL work, and the early development of DPL could not be carried out smoothly. In the 1970s, Conant and Reno's LD laterally pumped devices were still experimenting at low temperatures. Jackson and Rice used short pulses. The pump is quasi-continuously output. Farmer and Kiang have studied the lateral pumping of LEDs in a comprehensive way, and the end pumping with LD has also been reported. In the mid-1970s, A new concentration of quenching laser material with a higher doping concentration than Nd:YAG, but which does not cause the lifetime of the laser, has a wider range of choices as a solid gain material. One of the materials is Nd P504. (NPP). Experiments show that the threshold power of LD pump is $7 \mathrm{~m} \mathrm{~W}$. However, since its absorption peak (580nm) is not in the emission wavelength range of LD, its efficiency is only 7\%. Another material is Li Nd P4012. (LNP), when pumped with LD, it can get $1.05 \mu \mathrm{m}$ or $1.3 \mu \mathrm{m}$ laser output. Stone and Burrus first realized the waveguide fiber laser, the typical fiber core diameter is $35 \mu \mathrm{m}$, the length is $1 \mathrm{~cm}$. This waveguide laser uses LD Side pumping can be used as a fiber optic relay amplifier. The frequency doubling experiment performed in the DPL experiment is difficult because the cavity loss caused by the insertion of various optical components is too large, and the pump energy is too small. The power of LD is not a big breakthrough. The output power is near-infrared, and the highest is tens of milliwatts. The development of LD-pumped solid-state lasers is relatively slow, but DPL research is in waveguide lasers, new solid materials. Significant progress has been made in material development and unit technology research. 


\section{Yb : YAG all solid state laser}

In recent years, breakthroughs have been made in the preparation of transparent optical ceramic materials, and laser ceramics have developed rapidly as a new type of laser gain dielectric material. Yb: YAG laser ceramics are simple in manufacturing process and low in cost, and can realize multi-layer or multi-functional ceramic structure, and the absorption and emission spectra, fluorescence lifetime and thermal conductivity of ceramics are similar to those of single crystal. Therefore, high quality Yb:YAG ceramics will be a strong competitor for Yb:YAG single crystal materials in the future. In 2003, K. Takaichi et al. first reported LD-pumped Yb:YAG ceramic lasers. Adopt 2. The $4 \mathrm{~W}$ power pump doping concentration is 1 at. \%, thickness is 1 . A $6 \mathrm{~mm} \mathrm{Yb:YAG}$ ceramic with a $345 \mathrm{~mW} 1039 \mathrm{~nm}$ laser output. In 2006, Japan's JunDong et al. reported a Yb:YAG ceramic laser with passively Q-switched Cr:YAG ceramics, and obtained a $1030 \mathrm{~nm}$ laser with a peak power of $82 \mathrm{~kW}$ and a pulse width of 380 ps. In 2007, Wu Yusong and others from the Shanghai Silicate Research Institute realized the preparation of Yb:YAG ceramics for the first time in China, and obtained a $268 \mathrm{~mW} 1030 \mathrm{~nm}$ laser at a pump power of $7 \mathrm{~W}$. In the same year, Xu Yi and others at the Shanghai Institute of Optics and Mechanics used domestic Yb:YAG laser ceramics to obtain a pump power of 9 W. 63 W $1030 \mathrm{~nm}$ laser. In 2013, Cheng Ying et al. reported the results of studying the optical properties of $\mathrm{Cr}$, Yb:YAG self-adjusting Q-sheet lasers by bonding Yb:YAG laser ceramics. The pumping power is 7. Obtained 0 under $1 \mathrm{~W}$ conditions. $53 \mathrm{~W}$ self-adjusting $\mathrm{Q}$ laser output, light and light conversion efficiency is 7.5\%. A self-tuning Q pulse output with a pulse width of less than $3 \mathrm{~ns}$, a pulse energy of more than $25 \mu \mathrm{J}$, and a peak power of $9 \mathrm{~kW}$ was obtained in the experiment. In 2015, Wang Wei, Zhu Jiangfeng and others experimentally studied the high-efficiency continuous laser output and wavelength-tuned output characteristics of LD pumped domestic Yb:YAG ceramics. The output laser properties of laser ceramics with different atomic fractions (1\%, 2\%, 5\%, 10\%, 15\%) and different thicknesses (3 mm, $6 \mathrm{~mm}$ ) were compared. It fully proves the bright prospect of high-quality domestic Yb:YAG ceramics in high-power laser output, and verifies the feasibility of using ultra-short laser pulses by mode-locking technology. In 2016, Yu Yang and Zhu Jiangfeng realized the laser diode pumping of domestic Yb:YAG ceramic high-power passive Q-switched laser.

\section{Yb : YAG thin-film laser}

Thin-film lasers, as the name implies, have a very thin thickness of the gain medium. The thickness of the laser crystal is generally about several hundred micrometers, and the length of the cavity of the thin-film laser is very short. Because the thin-film laser crystal is thin and the cavity length is short, the cavity is made. The frequency spacing between the two longitudinal modes increases, and thus exceeds the gain bandwidth, that is, there is only one longitudinal mode within the width of the fluorescent line, so that single longitudinal mode laser oscillation is easily obtained. The LD pumped thin-film laser has the advantages of small size, light weight, simple structure, integrated optical system, etc., which is convenient for high-efficiency operation. It is favored in the material micro-machining industry. The LD-pumped thin-film laser has good single-frequency performance. Widely used in the storage of optical information, holography and other fields. In recent years, thin-film lasers have gradually emerged in the military field, and technologies such as ranging and guidance using lasers have been widely used. In 1994, Germany's Giesen et al. first designed a diode-pumped Yb:YAG thin-film laser with a multi-pass pump structure using Yb:YAG crystal as the laser gain medium. Since then, research on Yb:YAG thin-film lasers has developed rapidly. 2000, C. Stewen et al. used a Yb:YAG thin-film laser to achieve a continuous laser with a power of $647 \mathrm{~W}$. Based on this, the experiment was upgraded and a power output of $1070 \mathrm{~W}$ was obtained using four thin-film Yb:YAG. In 2003, the Swiss Union Institute of Technology realized the use of SESAM for mode-locking. Using the experimental setup shown in Figure 4, the pulse width of the beam near the diffraction limit was $810 \mathrm{fs}$, the output laser power was $60 \mathrm{~W}$, and the peak power was as high as 1. 9 MW pulse output. In 2004, Trumpf Laser of Germany adopted a method of placing multiple sheets in a cavity, that is, increasing the output power of the laser by 
scaling amplification, achieving a continuous laser output of $9 \mathrm{~kW}$. In 2006, the Swiss Federal Institute of Technology still used the Yb:YAG sheet gain medium to obtain a pulse width of $800 \mathrm{fs}$ and a peak power of 5. $6 \mathrm{MW} 1030 \mathrm{~nm}$ laser. In 2010, Trumpf Laser's thin-film lasers produced a maximum power of $16 \mathrm{~kW}$, a divergence angle of $8 \mathrm{mrad}$, and a laser power stability of greater than 99\%, which is sufficient for industrial needs. In 2011, Wang Chunhua of the China Institute of Engineering Physics and others used a 16-pass pumping system to obtain $27 \mathrm{~W}$ of continuous laser light with a light-to-light conversion efficiency of 38.8\%. In the same year, the Quantenoptik Institute in Germany realized a power-adjustable Yb:YAG thin-plate oscillator with Kerr lens mode-locking. In the experiment, an average power of $17 \mathrm{~W}$, a repetition rate of $40 \mathrm{MHz}$, and a light-to-light conversion efficiency were obtained. It is 25\%. In 2015, M. Hemmer et al. used a cryogenically cooled Yb:YAG thin-film laser with a pulse energy of up to $160 \mathrm{~mJ}$ to obtain an excellent laser beam with a repetition rate of $100 \mathrm{~Hz}$.

\section{Dual wavelength Yb: YAG laser}

Lasers are monochromatic, and a laser typically outputs only a single wavelength of laser while suppressing other wavelengths. However, in many fields, precision spectroscopic analysis, differential absorption laser radar, laser medical treatment, etc. require a laser capable of simultaneously outputting two or more wavelengths. In addition, by performing nonlinear mixing on multi-wavelength lasers, wavelengths in certain specific wavelength bands can be obtained, which is also important for the study of nonlinear optical mixing. The all-solid-state dual-wavelength laser has a small size, compact structure, and large coverage band, and has shown wide application prospects. In 2006, researchers such as Jun Dong realized the high-efficiency dual-wavelength optical properties of Yb:YAG crystals grown by temperature gradient technique at room temperature, demonstrating the best efficiency of laser pumped Yb:YAG crystals with wavelengths of $1030 \mathrm{~nm}$ and 1049. Nm, and achieves a slope efficiency of up to 68\% and a light-to-light conversion rate of more than 51\% at $1030 \mathrm{~nm}$. Experiments have shown that Yb:YAG crystals grown by temperature gradient techniques have very good optical properties and are good materials for high power dual wavelength lasers. In 2009, H. Yoshioka et al. designed a diode-pumped erbium-doped femtosecond laser with YAG ceramic as the main material, which was successfully implemented in $1033.5 \mathrm{~nm}$ and 1048. The passive mode at $3 \mathrm{~nm}$ is locked. This is the first mode-locked Yb:YAG ceramic laser and is the shortest pulse obtained by a diode-pumped ceramic laser without Kerr lens mode locking, but the mode-locking of the two wavelengths is not synchronized in this scheme. 2010, H. Yoshioka et al. improved the experimental scheme and successfully achieved 1033 in the laser. 6nm and 1047. The $6 \mathrm{~nm}$ two strongest gain lines simultaneously independently generate mode lock, at which point dual wavelength mode lock can be observed in one beam. The results show that the measurement of the double-wavelength lock mode can be 1033. $6 \mathrm{~nm}$ and 1047. Independently implemented between $6 \mathrm{~nm}$, this is the first time that a erbium-doped solid-state laser has achieved dual-wavelength mode-locking. In 2011, Yan Xinjie of Harbin Engineering University used $808 \mathrm{~nm}$ LD as the pump source pumped Nd: YVO4 crystal, and adopted Z-cavity to achieve effective oscillation of $914 \mathrm{~nm}$ fundamental light. The 915 $\mathrm{nm}$ absorption line of the Yb:YAG crystal was used to achieve $1030 \mathrm{~nm}$ photo-oscillation using 914 nm optically pumped Yb3+ doped 8\% Yb:YAG crystal. In 2013, Fengjiang Zhuang et al. proposed a continuous wave (CW) intracavity frequency-doubled Yb:YAG laser. The $4 \mathrm{~W} 1030 \mathrm{~nm}$ and $5.4 \mathrm{~W}$ $515 \mathrm{~nm}$ lasers are simultaneously output. The laser system is designed for photothermal common path interferometry to measure the spatially resolved profile of the linear absorption of visible or infrared light in the dielectric and coating, and the nonlinear absorption of the combination of the two.

\section{Conclusion}

In this paper, the advantages of Yb:YAG as a laser gain medium are analyzed. The research progress of laser diode-pumped Yb:YAG lasers at home and abroad is summarized. The Yb:YAG 
transparent ceramic lasers and erbium-doped fiber lasers are introduced. The latest research on $\mathrm{Yb}$ : YAG laser, Yb: YAG thin-film laser and dual-wavelength Yb: YAG laser, and its development prospects are prospected.

\section{References}

[1] Nakamura S, Yoshioka H, Yu M, et al. Efficient tunableYb : YAG ceramic laser [J] . Optics Communications, 2008, 281(17): 4411 - 4414.

[2] Stewen C, Contag K, Larinov M, et al. A 1 kW CW thindisk laser [J]. IEEE J. Quantum Electron, 2000, 6 (4): 650 - 657.

[3] E Innerhofer, T Südmeyer, et al. 60W average power in810fs pulses from a thin - disk Yb : YAG laser [J] . Opt. Lett., 2003, 28( 5) : 367 - 369.

[4] Sergio V. Marchese, Thomas Südmeyer, Matthias Golling, Rachel Grange and Ursula Keller. Pulse energy scaling to $5 \mu \mathrm{J}$ from a femtosecond thin disk laser [J]. Opt. Lett., 2006, 31(18) : 2728 2730.

[5] WANG Chunhua, WANG Weimin, et al. Design and experiment of multi-pass pump system for Yb: YAG thin- disk laser [J]. High Power Laser and Particle Beams, 2011, 23(5) : 1230 - 1232. 